\title{
Antibiotic Use and Need for Antimicrobial Stewardship in Long-Term Care: Correction
}

In an article about a study identifying potential antimicrobial stewardship needs in a long-term care facility, ${ }^{1}$ data for median days of therapy (DOT) were calculated incorrectly, which led to errors in the text and Table 1.

In the Results section, under the subheading "Antibiotic Use", the statement about median DOT should read as follows, with the corrected values shown in bold:

The median DOT per 1000 patient-days per month was 38.97 (interquartile range $[\mathrm{IQR}]$ 29.06-43.87) for all antibiotic routes combined (IV, oral, gastric tube, intraperitoneal, and topical),
6.93 (IQR 6.09-9.62) for IV antibiotics, and 27.63 (IQR 21.73-33.78) for oral antibiotics.

In addition, the data columns in Table 1 reporting median DOT/1000 patient-days were incorrect. In the complete table shown here, all values in the column for median DOT/1000 patient-days (and interquartile range) have been corrected.

Reference

1. Wu LDY, Walker SAN, Elligsen M, Palmay L, Simor A, Daneman N. Antibiotic use and need for antimicrobial stewardship in long-term care. Can J Hosp Pharm. 2015;68(6):445-9.

Table 1. Overall Antibiotic Use and Duration of Therapy over Study Period*

\begin{tabular}{|c|c|c|c|}
\hline \multirow{2}{*}{$\begin{array}{l}\text { Antibiotic Use } \\
\text { Route [heading corrected] }\end{array}$} & \multirow[t]{2}{*}{ No. of Orders } & \multicolumn{2}{|c|}{$\begin{array}{c}\text { Median DOT/1000 Patient-Days } \\
\text { (and IQR) } \\
\text { [all data values corrected] }\end{array}$} \\
\hline & & & \\
\hline Total antibiotics, any route & 835 & 38.97 & $(29.06-43.87)$ \\
\hline $\mathrm{PO}$ & 594 & 27.63 & $(21.73-33.78)$ \\
\hline IV & 208 & 6.93 & $(6.09-9.62)$ \\
\hline \multicolumn{4}{|l|}{$\begin{array}{l}\text { Total use, by class, subclass, } \\
\text { or individual drug }\end{array}$} \\
\hline Aminoglycosides & 10 & 0.00 & $(0.00-0.26)$ \\
\hline Carbapenems & 2 & 0.00 & $(0.00-0.07)$ \\
\hline Cephalosporins & 251 & 9.82 & $(7.14-13.85)$ \\
\hline First-generation & 81 & 3.41 & $(2.47-5.29)$ \\
\hline Second-generation & 54 & 2.04 & $(1.40-3.27)$ \\
\hline Third-generation & 116 & 4.41 & $(3.17-5.05)$ \\
\hline Clindamycin & 15 & 0.30 & $(0.03-1.79)$ \\
\hline Fluoroquinolones & 235 & 9.62 & $(7.84-13.18)$ \\
\hline Ciprofloxacin & 127 & 4.89 & $(3.50-8.21)$ \\
\hline Levofloxacin & 105 & 3.72 & $(3.38-5.02)$ \\
\hline Moxifloxacin & 3 & 0.00 & $(0.00-0.14)$ \\
\hline Macrolides & 56 & 0.90 & $(0.38-1.40)$ \\
\hline Metronidazole & 43 & 1.92 & $(1.05-2.83)$ \\
\hline Nitrofurantoin & 39 & 1.90 & $(1.50-2.26)$ \\
\hline Penicillins & 103 & 4.42 & $(3.62-6.58)$ \\
\hline Amoxicillin & 49 & 1.75 & $(1.24-2.39)$ \\
\hline Amoxicillin-clavulanate & 7 & 0.11 & $(0.00-1.33)$ \\
\hline Ampicillin & 9 & 0.04 & $(0.00-0.35)$ \\
\hline Cloxacillin & 20 & 0.77 & $(0.22-2.28)$ \\
\hline Penicillin & 1 & 0.00 & $(0.00-0.00)$ \\
\hline Piperacillin-tazobactam & 17 & 0.37 & $(0.12-0.88)$ \\
\hline Rifampin & 4 & 0.00 & $(0.00-0.14)$ \\
\hline Sulfamethoxazole-trimethoprim & 41 & 2.79 & $(1.69-3.30)$ \\
\hline Tetracyclines & 16 & 0.85 & $(0.00-1.93)$ \\
\hline Vancomycin IV & 4 & 0.00 & $(0.00-0.17)$ \\
\hline Vancomycin PO & 16 & 0.37 & $(0.02-0.67)$ \\
\hline \multicolumn{4}{|c|}{$\begin{array}{l}\text { DOT = days of therapy, IQR = interquartile range, IV = intravenous, } \mathrm{PO}=\text { by mouth. } \\
\text { *Study period was April 1, 2011, to March } 31,2012 \text { (12 months), and the total number of } \\
\text { patient-days was } 162 \text { 080, with an average of } \mathbf{1 3} \mathbf{5 0 7} \text { patient days per month used for } \\
\text { monthly (12 data points, } \mathbf{1} \text { data point per month) DOT/1000 patient-days statistics } \\
\text { [addition to footnote shown in bold]. } \\
\text { tRoutes of administration: IV, oral, gastric tube, intraperitoneal, and topical. }\end{array}$} \\
\hline
\end{tabular}

